\title{
Biologia comparada de Spodoptera cosmioides (Walk.) (Lepidoptera: Noctuidae) em cebola, mamona, soja e feijã $0^{1}$
}

\author{
Compared biology of Spodoptera cosmioides (Walk.) (Lepidoptera: Noctuidae) in onion, \\ castor oil plant, soybean and bean
}

\author{
Alvimar Bavaresco ${ }^{2}$ Mauro Silveira Garcia ${ }^{3}$ Anderson Dionei Grützmacher ${ }^{4}$ \\ Josemar Foresti ${ }^{5}$ Rudiney Ringenberg ${ }^{5}$
}

RESUMO

Estudou-se a biologia de Spodoptera cosmioides (Walk.) sobre quatro hospedeiros naturais (folhas de cebola, mamona, soja cv. Embrapa 48 e feijão cv. Carioca). Foram avaliados a duração e viabilidade das fases imaturas e do ciclo total, número de ínstares, peso de pupas, razão sexual, porcentagem de deformação de adultos e de adultos não liberados dos invólucros pupais, longevidade de adultos, fecundidade e viabilidade de ovos. Observou-se menor duração e maior viabilidade do periodo ovo-adulto para mamona e cebola (39,7 dias e 21,7\% e 40,5 dias e 21,8\%, respectivamente), do que para soja (46,0 dias e 3,9\%). O peso pupal foi de 444,6 $\mathrm{mg}$ em cebola, 420,0 em feijão, 396,6 em mamona e 298,7 em soja. Constatouse um predomínio de seis instares larvais em folhas de cebola e mamona, ocorrendo também sete para alguns individuos, enquanto que em soja predominou a ocorrência de sete, também verificando oito instares, indicando uma menor adequação nutricional deste hospedeiro. As fêmeas apresentaram duração da fase pupal significativamente menor do que os machos em todos hospedeiros, fazendo com que a emergência do adulto ocorresse antes dos machos. Em cebola, a longevidade de adultos foi significativamente superior aos demais hospedeiros. A fecundidade foi maior em cebola e mamona (3224 e 3206 ovos/ fêmea, respectivamente), enquanto que para soja observou-se 1353 ovos/fêmea. Em folhas de feijão, o desenvolvimento do inseto foi prejudicado por caracteres morfológicos - pilosidade, que promoveram elevada mortalidade na fase larval.

Palavras-chave: insecta, bioecologia, nutrição de insetos.

\begin{abstract}
The biology of Spodoptera cosmioides (Walk.) was studied on four natural hosts (onion leaves, castor oil plant, soy cv. Embrapa 48 and bean cv. Carioca). The duration and viability of immature phases and total cycle, instar number, pupals weight, sexual ratio, percentage of deformed adults and adults not released of pupal beg, adults' longevity, fecundity and eggs viability were evaluated. It was observed in castor oil plant and onion shorter duration and larger viability of total cycle (39.7 days and 21.7\%; 40.5 days and $21.8 \%$, respectively), in contrast of soy (46.0 days and 3.9\%). The pupal weight was $444.6 \mathrm{mg}$ on onion, $420.0 \mathrm{mg}$ on bean, $396.6 \mathrm{mg}$ on castor oil plant and 298.7 mg on soy. Onion and castor oil plant leaves had a predominance of six larval instars, also happening seven for some of them, while in soy the occurrence of seven prevailed, also happening eight instars, indicating less nutritional adaptation of this host. The females had pupal phase significantly smaller than the males in all the hosts, making adults emergency happen before the males. In onion, the longevity of the adults was significantly superior comparing with the other hosts. The fecundity was better on onion and castor oil plant (3224 and 3206 eggs/ female, respectively), therefore on soy was observed 1353 eggs/female. In bean leaves, the development of the insect was harmed by morphologic characters, which promoted high mortality in the larval phase.
\end{abstract}

Key words: insecta, bioecology, nutrition of insects.

${ }^{1}$ Parte da dissertação apresentada pelo primeiro autor ao Programa de Pós-graduação (PPG) em Fitossanidade / Entomologia, Faculdade de Agronomia "Eliseu Maciel" (FAEM), Universidade Federal de Pelotas (UFPel).

${ }^{2}$ Engenheiro Agrônomo, Mestre, Pesquisador, Epagri. BR 280, Km 219,5, Bairro Campo da Água Verde, CP 216, 89460-000, Canoinhas, SC. E-mail: bavaresco@epagri.rct-sc.br. Autor para correspondência.

${ }^{3}$ Engenheiro Agrônomo, Doutor, Professor Adjunto, Departamento de Fitossanidade, FAEM/UFPel. Campus Universitário s/n. CP 354, Pelotas, RS. E-mail: msgarcia@ufpel.tche.br.

${ }^{4}$ Engenheiro Agrônomo, Doutor, Professor Adjunto, Departamento de Fitossanidade, FAEM/UFPel. E-mail: adgrutz@ufpel.tche.br.

${ }^{5}$ Engenheiro Agrônomo, Aluno do PPG em Fitossanidade/Entomologia (Mestrado), FAEM/UFPel. 


\section{INTRODUÇÃO}

Spodoptera cosmioides (Walk.) é uma espécie polífaga. No Brasil, diversas culturas são citadas como suas hospedeiras, entre as quais o algodoeiro, o pimentão, o tomateiro, a mamona, o feijão o caupi, o eucalipto, o abacaxizeiro, o arroz, a cebola, a mangueira, a berinjela, entre outras (BERTELS, 1953; SILVA et al., 1968; SANTOS et al., 1980; GALLO et al., 2002). Entretanto, apesar da ampla gama de hospedeiros, sua ocorrência como praga é relatada em apenas algumas culturas, e geralmente relacionada a desequilíbrios provocados pelo uso excessivo de inseticidas de amplo espectro. Nos Estados de São Paulo, Paraná e Santa Catarina, ocorreram surtos da praga, causando severos danos em culturas de interesse econômico (HABIB et al., 1983). NORA \& REIS FILHO (1988) relataram danos elevados de $\boldsymbol{S}$. cosmioides e $\boldsymbol{S}$. eridania em frutos e folhas de macieira no Estado de Santa Catarina. Estas duas espécies também são as principais lagartas que atacam vagens de soja, assumindo importância a partir do início da fase reprodutiva da cultura (GAZZONI \& YORINORI, 1995; REUNIÃODE PESQUISA DE SOJA DA REGIÃO SUL, 2000).

S. cosmioides era até pouco tempo considerada sinonímia de Spodoptera latifascia (Walk.). Entretanto, diferenças moleculares, morfológicas, fisiológicas e comportamentais fazem com que devam ser consideradas espécies distintas (SILVAIN \& LALANNE-CASSOU, 1997; LALANNE-CASSOU et al., 1999). Ambas são membros de um complexo de espécies neotropicais, sendo que $\boldsymbol{S}$. latifascia encontra-se estabelecida na América Central, Antilhas e Sul do Estados Unidos, enquanto $S$. cosmioides é encontrada na América do Sul.

S. latifascia foi identificada como uma das espécies de um complexo de noctuídeos que causa severos danos em milho e sorgo em Honduras, durante o início do desenvolvimento da cultura (PITRE, 1988). Posteriormente, PORTILLO et al. (1991) verificaram que estas plantas não eram boas hospedeiras para o inseto, que não completou o ciclo de vida quando as lagartas foram alimentadas exclusivamente com milho ou sorgo. O inseto apresenta uma estreita relação com a vegetação não cultivada presente na área, sendo que se as práticas culturais não incluírem o controle químico de plantas daninhas, permitindo seu estabelecimento na lavoura, as lagartas não causarão maiores prejuízos às culturas citadas (PORTILLO et al., 1991; PORTILLO et al., 1996; PITRE et al., 1997).

No ano de 1999, S. cosmioides causou severos danos em áreas experimentais de cebola cultivada em casa de vegetação no Campus da Universidade Federal de Pelotas, RS. De acordo com PARRA (2000), o conhecimento da biologia de um inseto é de fundamental importância para se desenvolverem estratégias de manejo eficientes, dentro dos conceitos do manejo integrado de pragas.

Desta forma, o presente trabalho teve por objetivo avaliar a biologia de $\boldsymbol{S}$. cosmioides em folhas de cebola, comparada com mamona, soja e feijão, visando determinar os mais adequados ao seu desenvolvimento.

\section{MATERIAL E MÉTODOS}

O trabalho foi conduzido no laboratório de Biologia dos Insetos do Departamento de Fitossanidade, FAEM/UFPel, na temperatura de $26 \pm 1{ }^{\circ} \mathrm{C}$, UR de $70 \pm 10 \%$ e fotofase de 14 horas. Foi avaliada a biologia do inseto em quatro hospedeiros naturais: folhas de cebola, cultura na qual o inseto foi observado, mamona (plantas espontâneas), soja (cv. Embrapa 48) e feijão (cv. Carioca). Os parâmetros biológicos avaliados foram: duração e viabilidade das diferentes fases de desenvolvimento e do ciclo total; número de ínstares; peso de pupas; razão sexual; porcentagem de deformação de adultos; porcentagem de adultos não liberados dos invólucros pupais; longevidade de adultos (machos e fêmeas); fecundidade (diária e total) e viabilidade de ovos.

Foram utilizadas 150 lagartas recém eclodidas, provenientes da criação de manutenção em dieta artificial, as quais foram transferidas para tubos de vidro de $8,5 \mathrm{~cm} \times 2,5 \mathrm{~cm}$ tampados com algodão hidrófugo, contendo uma folha ou parte de uma folha do hospedeiro respectivo e um pedaço de papel filtro umedecido. Antes de serem fornecidas as lagartas, as folhas foram tratadas com solução de sulfato de cobre (1\%) durante um minuto, sendo em seguida mergulhadas em água destilada pelo mesmo período, e postas a secar à sobra por uma hora, visando eliminar entomopatógenos que pudessem contaminar as lagartas. Ao atingirem o último ínstar, as lagartas foram transferidas para copos plásticos de $7,3 \mathrm{~cm}$ de diâmetro x $5,3 \mathrm{~cm}$ de altura, em virtude dos tubos de vidro não comportarem mais a quantidade de alimento necessária para as mesmas, sendo a tampa perfurada para evitar a condensação de água no seu interior. As pupas foram transferidas para tubos de vidro de $8,5 \mathrm{~cm} \times 2,5 \mathrm{~cm}$, onde permaneceram até a emergência dos adultos. A pesagem das pupas foi feita 24 horas após a pupação, quando também foi feita a sexagem, baseado em BUTT \& CANTU (1962). A razão sexual foi calculada através da fórmula $\mathrm{rs}=\mathrm{n}^{\mathrm{o}}$ de fêmeas $/ \mathrm{n}^{\mathrm{o}}$ fêmeas $+\mathrm{n}^{\mathrm{o}}$ 
machos (SILVEIRA NETO et al., 1976). O número de ínstares foi determinado através do método da curva de distribuição de freqüência, descrito por PARRA \& HADDAD (1989). Para esta determinação foi realizada a medição diária da cápsula cefálica de 40 lagartas, utilizando-se um microscópico estereoscópico equipado com ocular micrométrica.

Para o estudo da fecundidade e longevidade, individualizaram-se 20 casais de adultos em gaiolas de PVC de $15 \mathrm{~cm}$ de altura $x 15 \mathrm{~cm}$ de diâmetro revestidas internamente com papel jornal, dispostas em pratos plásticos também forrados com o mesmo papel, e fechadas na extremidade superior com tecido tipo tule, preso com elástico. Os adultos foram alimentados com solução de mel (10\%), fornecida por capilaridade através de algodão mantido em recipientes de vidro, renovado a cada dois dias. Diariamente observou-se a mortalidade de adultos e procedeu-se a retirada das posturas para contagem do número de ovos. Para avaliação da duração do período embrionário e viabilidade de ovos, diariamente foi incubada uma postura de cada casal, contendo 150 a 300 ovos, observando-se a porcentagem de ovos viáveis e o período de incubação dos mesmos. Estas posturas foram mantidas em tubos de vidro de $8,5 \mathrm{~cm} \times 2,5 \mathrm{~cm}$, fechados com filme plástico de PVC. A partir destes dados, foram calculadas as tabelas de vida de fertilidade em cada hospedeiro, baseando-se em SILVEIRA NETO et al. (1976).

O delineamento experimental utilizado foi o inteiramente casualizado. Para as avaliações relativas às fases jovens, foram utilizadas 30 repetições, sendo a unidade experimental formada por cinco indivíduos.
Para a fase adulta, utilizaram-se 20 repetições para cebola e mamona e 11 para soja, sendo cada unidade experimental composta por um casal. Os dados de cada parâmetro foram analisados quanto à homocedasticidade, transformados em $1 / \mathrm{x}^{1 / 2}$ (longevidade de fêmeas) e em $(x+0,5)^{1 / 2}$ (viabilidade do ciclo total), e analisados pelo teste $\mathrm{F}$ para análise da variância. As médias foram comparadas pelos testes de Tukey para os dados que apresentaram distribuição normal, ou de Kruskal-Wallis para os que não apresentaram, em nível de 5\% de probabilidade.

\section{RESULTADOS E DISCUSSÃO}

A duração da fase larval variou de 20,2 a 28,0 dias para lagartas criadas em folhas de mamona e soja, respectivamente (Tabela 1). A viabilidade larval foi mais elevada para mamona e cebola, sendo intermediária para soja e menor para feijão (Tabela 2). HABIB et al. (1983) observaram uma duração menor para a fase larval, 13,4 e 16,9 dias para algodoeiro e soja, respectivamente, na faixa de temperatura de 22,3 a $30,4^{\circ} \mathrm{C}$, e viabilidade de 95 e $92 \%$ para algodoeiro e soja, respectivamente. Neste trabalho, quando as lagartas foram criadas em folhas de soja e feijão, observou-se a ocorrência de um agente patogênico não identificado, que ocasionou mortalidade de 27 e $10 \%$, respectivamente. O patógeno deixava as lagartas com uma coloração escura e o corpo flácido que se rompia com um leve toque. A baixa viabilidade observada em folhas de feijão deveuse ao fato deste apresentar alta densidade de pêlos em

Tabela 1 - Duração (em dias) dos estágios de ovo, larva, pré-pupa, pupa e ciclo total, número de ínstares (n), razão de crescimento (K) e coeficiente de determinação $\left(\mathrm{R}^{2}\right)$ e peso de pupas em mg $(\mathrm{X} \pm \mathrm{EP})$ de Spodoptera cosmioides criadas em hospedeiros naturais. Temperatura: $26 \pm 1{ }^{\circ} \mathrm{C}$; UR: $70 \pm 12 \%$; fotofase: $14 \mathrm{~h}$

\begin{tabular}{|c|c|c|c|c|c|c|c|c|c|}
\hline \multirow{2}{*}{ Hosp. } & \multicolumn{5}{|c|}{ Duração dos estágios (dias) } & \multicolumn{3}{|c|}{ Ínstares } & \multirow{2}{*}{ Peso de pupas (mg) } \\
\hline & $\mathrm{Ovo}^{1}$ & Larva $^{2}$ & Pré-pupa ${ }^{1}$ & Pupa $^{1}$ & Ciclo total $^{1}$ & $\mathrm{~N}$ & $\mathrm{~K}$ & $\mathrm{R}^{2}$ & \\
\hline Mamona & $\begin{array}{c}4,12 \pm 0,04 \mathrm{a} \\
(3-5)[78]\end{array}$ & $\begin{array}{c}20,2 \pm 0,26 \mathrm{c} \\
(16-32)[116]\end{array}$ & $\begin{array}{l}2,7 \pm 0,11 \mathrm{a} \\
(1-5)[109]\end{array}$ & $\begin{array}{l}13,5 \pm 0,22 \mathrm{a} \\
(11-17)[70]\end{array}$ & $\begin{array}{l}39,7 \pm 0,51 b \\
(35-47)[70]\end{array}$ & $\begin{array}{l}6[25] \\
7[25]\end{array}$ & $\begin{array}{l}1,63 \\
1,45\end{array}$ & $\begin{array}{l}0,99 \\
0,99\end{array}$ & $\begin{array}{c}396,6 \pm 7,62 b \\
(200-590)[109]\end{array}$ \\
\hline Cebola & $\begin{array}{c}4,05 \pm 0,05 a \\
(3-5)[53]\end{array}$ & $\begin{array}{c}22,3 \pm 0,36 b \\
(17-34)[111]\end{array}$ & $\begin{array}{l}1,9 \pm 0,07 b \\
(1-3)[106]\end{array}$ & $\begin{array}{l}12,5 \pm 0,20 b \\
(10-15)[74]\end{array}$ & $\begin{array}{l}40,5 \pm 0,48 b \\
(36-53)[74]\end{array}$ & $\begin{array}{l}6[33] \\
7[33]\end{array}$ & $\begin{array}{l}1,67 \\
1,48\end{array}$ & $\begin{array}{l}0,99 \\
1,00\end{array}$ & $\begin{array}{c}444,6 \pm 4,51 \mathrm{a} \\
(280-550)[105]\end{array}$ \\
\hline Soja & $\begin{array}{c}4,11 \pm 0,06 \mathrm{a} \\
(4-5)[23]\end{array}$ & $\begin{array}{l}28,0 \pm 0,50 \mathrm{a} \\
(23-36)[68]\end{array}$ & $\begin{array}{c}2,4 \pm 0,09 a b \\
(1-4)[54]\end{array}$ & $\begin{array}{l}12,5 \pm 0,31 b \\
(10-14)[24]\end{array}$ & $\begin{array}{l}46,0 \pm 0,94 \mathrm{a} \\
(40-54)[24]\end{array}$ & $\begin{array}{l}7[25] \\
8[25]\end{array}$ & $\begin{array}{l}1,50 \\
1,38\end{array}$ & $\begin{array}{l}1,00 \\
1,00\end{array}$ & $\begin{array}{c}298,7 \pm 7,38 c \\
(200-410)[54]\end{array}$ \\
\hline Feijão & - & $\begin{array}{c}21,3 \pm 0,31 b c \\
(20-23)[8]\end{array}$ & $\begin{array}{c}1,7 \pm 0,33 b \\
(1-2)[3]\end{array}$ & $\begin{array}{c}11,0 \pm 1,00 b \\
(10-12)[2]\end{array}$ & - & $\begin{array}{l}- \\
-\end{array}$ & - & $\begin{array}{l}- \\
-\end{array}$ & $\begin{array}{c}420,0 \pm 32,15 \mathrm{ab} \\
(360-470)[3]\end{array}$ \\
\hline
\end{tabular}

${ }^{1}$ Médias não seguidas de mesma letra diferem estatisticamente entre si pelo teste de Tukey em $5 \%$ de probabilidade;

${ }^{2}$ Médias não seguidas de mesma letra diferem estatisticamente entre si pelo teste de Kruskall-Wallis em 5\% de probabilidade;

Valores entre parênteses expressam o intervalo de variação e entre colchetes o número de observações [n]. 
Tabela 2 - Viabilidade (\%) dos estádios de ovo, larva, pré-pupa, pupa e ciclo total (X \pm EP) e razão sexual de Spodoptera cosmioides criada em hospedeiros naturais. Temperatura: $26 \pm 1^{\circ} \mathrm{C}$; UR: $70 \pm 12 \%$; fotofase: $14 \mathrm{~h}$.

\begin{tabular}{|c|c|c|c|c|c|c|}
\hline \multirow{2}{*}{ Hospedeiros } & \multicolumn{5}{|c|}{ Viabilidade dos estádios (\%) } & \multirow{2}{*}{ Razão Sexual } \\
\hline & $\mathrm{Ovo}^{1}$ & Larva $^{1}$ & Pré-pupa ${ }^{1}$ & Pupa $^{1}$ & Ciclo total $^{2}$ & \\
\hline Mamona & $\begin{array}{c}46,3 \pm 4,35 \mathrm{a} \\
{[83]}\end{array}$ & $\begin{array}{c}77,3 \pm 3,42 \mathrm{a} \\
{[150]}\end{array}$ & $\begin{array}{c}93,1 \pm 2,48 \mathrm{a} \\
{[116]}\end{array}$ & $\begin{array}{c}65,0 \pm 4,44 \mathrm{a} \\
{[109]}\end{array}$ & $\begin{array}{c}21,7 \pm 2,98 \mathrm{a} \\
{[150]}\end{array}$ & 0,46 \\
\hline Cebola & $\begin{array}{c}42,9 \pm 7,00 \mathrm{a} \\
{[68]}\end{array}$ & $\begin{array}{c}74,0 \pm 4,20 \mathrm{a} \\
{[150]}\end{array}$ & $\begin{array}{c}93,0 \pm 3,68 \mathrm{a} \\
{[111]}\end{array}$ & $\begin{array}{c}73,7 \pm 4,67 \mathrm{a} \\
{[106]}\end{array}$ & $\begin{array}{c}21,8 \pm 2,76 \mathrm{a} \\
{[150]}\end{array}$ & 0,48 \\
\hline Soja & $\begin{array}{c}24,7 \pm 4,41 \mathrm{a} \\
{[28]}\end{array}$ & $\begin{array}{c}45,3 \pm 4,49 b \\
{[150]}\end{array}$ & $\begin{array}{c}73,6 \pm 6,52 \mathrm{ab} \\
{[68]}\end{array}$ & $\begin{array}{c}47,3 \pm 7,96 \mathrm{a} \\
{[54]}\end{array}$ & $\begin{array}{c}3,9 \pm 2,35 \mathrm{~b} \\
{[150]}\end{array}$ & 0,31 \\
\hline Feijão & - & $\begin{array}{c}5,3 \pm 1,64 \mathrm{c} \\
{[150]}\end{array}$ & $\begin{array}{c}37,5 \pm 18,30 \mathrm{~b} \\
{[8]}\end{array}$ & $\begin{array}{c}65,0 \pm 33,33 \mathrm{a} \\
{[3]}\end{array}$ & - & - \\
\hline
\end{tabular}

${ }^{1}$ Médias não seguidas de mesma letra diferem estatisticamente entre si pelo teste de Kruskal-Wallis em 5\% de probabilidade;

${ }^{2}$ Médias não seguidas de mesma letra diferem estatisticamente entre si pelo teste de Tukey em 5\% de probabilidade;

Valores entre colchetes expressam o número de observações [n].

forma de gancho, os quais se prendiam aos pseudópodes das lagartas, impedindo sua locomoção e ocasionando, conseqüentemente, a sua morte. A eficiência dos pêlos em aprisionar as lagartas foi maior nos primeiros ínstares, sendo que as que atingiam os últimos ínstares não eram mais afetadas. As folhas de soja também apresentam densa pilosidade, porém estes são longos e eretos, não aprisionando os insetos. LARA (1991) relatou que tricomas normais podem atuar diretamente sobre os indivíduos, afetando sua oviposição, alimentação, locomoção ou seu comportamento em relação ao abrigo, dependendo de sua densidade e tamanho.

Em folhas de mamona e cebola, as lagartas apresentaram seis ou sete ínstares, sendo 56 e $44 \%$ da população para mamona e 55 e $46 \%$ para cebola, respectivamente. Em folhas de soja predominou a ocorrência de sete ínstares, em $86 \%$ da população, com os demais $16 \%$ apresentando oito ínstares (Tabela 1). O maior número de ínstares em folhas de soja indica uma menor adequação deste alimento para o desenvolvimento das lagartas. SANTOS et al. (1980) também observaram seis a sete ínstares para lagartas alimentadas com folhas de eucalipto, enquanto HABIB et al. (1983) verificaram a ocorrência de cinco ínstares em média ( $69 \%$ da população) em folhas de algodoeiro, ocorrendo também quatro e seis ínstares, e em média seis ínstares ( $54 \%$ da população) em folhas de soja, com variação de quatro a oito. No caso da soja, deve-se levar em consideração a preferência da praga por alimentar-se de vagens em relação a folhas (GAZZONI \& YORINORI, 1995), podendo as vagens serem mais adequadas para o seu desenvolvimento.
A duração da fase pré-pupal variou de 1,7 a 2,7 dias em feijão e mamona, respectivamente (Tabela 1). A viabilidade foi superior a $90 \%$ para mamona e cebola, sendo bastante reduzida $(38 \%)$ em feijão (Tabela 2). A baixa viabilidade neste hospedeiro pode não ser representativa, devido ao baixo número de indivíduos que atingiram esta fase, de modo que a morte de poucos indivíduos representou uma grande variação na viabilidade. HABIB et al. (1983) observaram um período pré-pupal de aproximadamente 1,4 dias, tanto para machos como para fêmeas na faixa de temperatura de 22,3 a $30,4^{\circ} \mathrm{C}$, quando as lagartas foram alimentadas com folhas de soja. Em algodoeiro este período foi de 1,4 dias para fêmeas e 1,3 dias para machos.

A fase pupal variou de 11,0 a 13,5 dias em feijão e mamona, respectivamente, sendo significativamente superior somente em mamona (Tabela 1). SANTOS et al. (1980) obtiveram resultados semelhantes para lagartas alimentadas com folhas de eucalipto, sob temperatura média de $22,3^{\circ} \mathrm{C}$ e umidade relativa de $76,6 \%$. Entretanto, os resultados de HABIB et al. (1983), que observaram durações de 9,5 e 11,4 dias para algodoeiro e soja, respectivamente, diferem dos observados no presente experimento. A viabilidade pupal foi maior em cebola (74\%) e menor em soja (47\%) (Tabela 2). No caso do feijão, deve-se considerar as estruturas morfológicas presentes na cultivar, não sendo possível afirmar que esta espécie vegetal é nutricionalmente inadequada para $\boldsymbol{S}$. cosmioides. O peso médio de pupas variou de $298,7 \mathrm{mg}$ a 444,6mg em soja e cebola, respectivamente (Tabela

Ciência Rural, v. 33, n. 6, nov-dez, 2003. 
1), sendo superior ao observado por HABIB et al. (1983), de 249 e 146mg para lagartas criadas em algodoeiro e soja, respectivamente. A razão sexual foi próxima a 0,5 para mamona e cebola, sendo menor em soja (Tabela 2).

A duração do ciclo total (ovo à emergência do adulto) foi de 46,0 dias em soja, 40,5 dias em cebola e 39,7 dias em mamona, não havendo diferença significativa entre os dois últimos (Tabela 1). Estes resultados são inferiores aos de SANTOS et al. (1980), que verificaram valores de 49,7 dias para o ciclo total em folhas de eucalipto. A viabilidade do ciclo total foi baixa para todos os hospedeiros (Tabela 2), principalmente em virtude da baixa viabilidade do período embrionário, sendo esta devida, provavelmente, a picos de baixa umidade do ar.

Tanto machos como fêmeas adultos foram mais longevos quando criados em folhas de cebola na fase larval (Tabela 3 ). O percentual de adultos deformados foi superior a $20 \%$ em soja e mamona, hospedeiros em que também foi observado um maior percentual de adultos que não conseguiram liberar-se totalmente dos invólucros pupais, indicando uma menor adequação nutricional destes (Tabela 3). Em feijão, este resultado ficou comprometido em função dos caracteres morfológicos da cultivar, sendo que apenas dois indivíduos atingiram a fase adulta, e um apresentou deformações. SANTOS et al. (1980) encontraram longevidades inferiores, de 8,7 e 6,4 dias para fêmeas e machos, respectivamente, quando as lagartas foram criadas em folhas de eucalipto, sob temperatura de $22,3^{\circ} \mathrm{C}$ e umidade relativa de $76,6 \%$, enquanto que HABIB et al. (1983) observaram uma longevidade média de 13,2 e 12,3 dias quando as lagartas foram alimentadas com folhas de algodoeiro e soja, respectivamente, sob temperatura de 22,3 a $30,4^{\circ} \mathrm{C}$ e umidade relativa de $69,8 \%$.

A fecundidade total foi semelhante em mamona e cebola, sendo significativamente inferior em soja (Tabela 3). A fecundidade diária apresentou comportamento semelhante, porém sem diferir significativamente entre os três hospedeiros, apesar de ser bem menor em soja. SANTOS et al. (1980) observaram uma fecundidade média de 2016 ovos, quando as lagartas foram alimentadas com folhas de eucalipto, valores estes intermediários aos observados entre soja e os outros dois hospedeiros estudados neste trabalho. HABIB et al. (1983) afirmaram haver diferença no período de oviposição, fecundidade diária e total quando as lagartas foram criadas em folhas de soja e algodoeiro. Neste trabalho, a viabilidade de ovos foi inferior em soja, porém sem diferir significativamente de cebola e mamona (Tabela 2). A duração do período embrionário não apresentou variação em função do hospedeiro (Tabela 1).

O cálculo de tabela de vida de fertilidade foi feito somente para mamona e cebola, pois em soja e feijão o número de insetos que atingiram a fase adulta foi insuficiente para a avaliação. A taxa líquida de reprodução (Ro) foi superior em cebola, indicando maior adequação deste hospedeiro para o desenvolvimento do inseto. Os demais resultados

Tabela 3 - Longevidade de adultos (em dias), fecundidade diária e total (X \pm EP), deformação de adultos (da) e adultos não liberados totalmente dos invólucros pupais (anl), duração média de uma geração (T), taxa líquida de reprodução (Ro), razão infinitesimal de aumento (rm) e razão finita de aumento $(\lambda)$ de Spodoptera cosmioides em hospedeiros naturais. Temperatura: $26 \pm 1{ }^{\circ} \mathrm{C}$; UR: $70 \pm 12 \%$; fotofase: $14 \mathrm{~h}$

\begin{tabular}{|c|c|c|c|c|c|c|c|c|c|c|}
\hline \multirow{2}{*}{ Hosp. } & \multicolumn{2}{|c|}{ Longevidade } & \multirow{2}{*}{$\begin{array}{c}\mathrm{da} \\
(\%)\end{array}$} & \multirow{2}{*}{$\begin{array}{l}\text { Anl } \\
(\%)\end{array}$} & \multicolumn{2}{|c|}{ Fecundidade } & \multirow{2}{*}{$\begin{array}{c}\mathrm{T} \\
\text { (dias) }\end{array}$} & \multirow{2}{*}{ Ro } & \multirow{2}{*}{$\mathrm{Rm}$} & \multirow{2}{*}{$\lambda$} \\
\hline & Machos $^{1}$ & Fêmeas $^{2}$ & & & Diária $^{2}$ & Total $^{1}$ & & & & \\
\hline Mamona & $\begin{array}{c}14,5 \pm 1,11 b \\
(8-27)[20]\end{array}$ & $\begin{array}{c}11,5 \pm 0,64 b \\
(7-18)[20]\end{array}$ & $\begin{array}{l}25,0 \\
{[70]}\end{array}$ & $\begin{array}{l}14,3 \\
{[70]}\end{array}$ & $\begin{array}{c}446,0 \pm 54,30 \mathrm{a} \\
(83,9-1016,2)[20]\end{array}$ & $\begin{array}{l}3205,6 \pm 426,80 a \\
(214-6537)[20]\end{array}$ & 47,19 & 313,61 & 0,12180 & 1,12953 \\
\hline Cebola & $\begin{array}{c}22,4 \pm 1,26 a \\
(9-31)[20]\end{array}$ & $\begin{array}{c}15,2 \pm 0,98 a \\
(9-25)[20]\end{array}$ & $\begin{array}{c}1,4 \\
{[74]}\end{array}$ & $\begin{array}{c}1,4 \\
{[74]}\end{array}$ & $\begin{array}{c}416,4 \pm 46,65 \mathrm{a} \\
(55,0-888,0)[20]\end{array}$ & $\begin{array}{l}3224,4 \pm 304,04 a \\
(165-6074)[20]\end{array}$ & 50,71 & 380,69 & 0,11718 & 1,12432 \\
\hline Soja & $\begin{array}{l}11,2 \pm 0,91 b \\
(7-17)[11]\end{array}$ & $\begin{array}{c}10,5 \pm 0,73 b \\
(5-13)[11]\end{array}$ & $\begin{array}{l}20,8 \\
{[24]}\end{array}$ & $\begin{array}{c}8,3 \\
{[24]}\end{array}$ & $\begin{array}{c}288,3 \pm 47,88 \mathrm{a} \\
(75,8-639,8)[11]\end{array}$ & $\begin{array}{l}1352,7 \pm 283,74 b \\
(379-2838)[11]\end{array}$ & - & - & - & - \\
\hline Feijão & - & - & $\begin{array}{c}50,0 \\
{[2]}\end{array}$ & $\begin{array}{l}0,0 \\
{[2]}\end{array}$ & - & - & - & - & - & - \\
\hline
\end{tabular}

${ }^{1}$ Médias não seguidas de mesma letra diferem estatisticamente entre si pelo teste de Kruskal-Wallis em 5\% de probabilidade; ${ }^{2}$ Médias não seguidas de mesma letra diferem estatisticamente entre si pelo teste de Tukey em 5\% de probabilidade;

Valores entre parênteses expressam o intervalo de variação e entre colchetes o número de observações [n]. 
observados foram semelhantes entre os dois hospedeiros, sendo a duração média de uma geração (T), ligeiramente maior em cebola (Tabela 3 ).

\section{CONCLUSÕES}

Folhas de cebola e de mamona constituem hospedeiros mais adequados ao desenvolvimento de S. cosmioides, observando-se menor duração e maior viabilidade do período ovo-adulto e maior peso pupal do que para folhas de soja.

Folhas de mamona tiveram comportamento intermediário no desenvolvimento do inseto, podendo esta espécie atuar como hospedeiro alternativo da praga nas condições do Rio Grande do Sul.

O feijoeiro cultivar Carioca impediu o desenvolvimento de $\boldsymbol{S}$. cosmioides em função da presença de estruturas morfológica (pêlos em forma de gancho) nas folhas, nas quais as lagartas ficam aprisionadas através dos pseudópodes.

\section{AGRADECIMENTOS}

À Coordenação de Aperfeiçoamento de Pessoal de Nível Superior (CAPES), pela concessão da bolsa durante o curso de Mestrado e à Fundação de Amparo a Pesquisa do Estado do Rio Grande do Sul (FAPERGS) pelo suporte financeiro.

\section{REFERÊNCIAS BIBLIOGRÁFICAS}

BERTELS, A. Pragas de solanáceas cultivadas. Agros, Pelotas, v.6, n.4, p.154-160, 1953.

BUTT, B.A.; CANTU, E. Sex determination of lepidopterous pupae. Washington : United States Department of Agriculture (USDA), Agricultural Research Service, 1962. 7p. (ARS-33-75).

GALLO, D. et al. Entomologia agrícola. Piracicaba : FEALQ, 2002. 920p.

GAZZONI, D.L.; YORINORI, J.T. Manual de identificação de pragas e doenças da soja. Brasília : EMBRAPA - SPI, 1995. 128p. (Manuais de Identificação de Pragas e Doenças, 1).

HABIB, M.E.M.; PALEARI, L.M.; AMARAL, M.E.C. Effect of three larval diets on the development of the armyworm, Spodoptera latifascia Walk., 1856 (Noctuidae, Lepidoptera). Revista Brasileira de Zoologia, São Paulo, v.1, n.3, p.177$182,1983$.

LALANNE-CASSOU, B. et al. Mecanismes d'isolement reproducteur chez les especes du complexe neotropical
Spodoptera latifascia-S. cosmioides-S. descoinsi (Lepidoptera: Noctuidae). Annales de la Societe Entomologique de France, v.35, Supp., p.109-116, 1999.

LARA, F.M. Princípios de resistência de plantas a insetos. São Paulo : Ícone, 1991. 336p.

NORA, I.; REIS FILHO, W. Damage to apple (Malus domestica, Bork.) caused by Spodoptera spp. (Lepidoptera: Noctuidae). Acta Horticulturae, v.232, p.209-212, 1988.

PARRA, J.R.P.; HADDAD. M.L. Determinação do número de ínstares de insetos. Piracicaba : FEALQ, 1989. 49p.

PARRA, J.R.P. A biologia de insetos e o manejo de pragas: da criação em laboratório à aplicação em campo. In: GUEDES J.V.C.; COSTA, I.D. da.; CASTIGLIONI, E. Bases e técnicas do manejo de insetos. Santa Maria : UFSM/CCR/DFS, 2000. Cap.1, p.129.

PITRE, H.N. A complex of lepidopterous defoliators on sorghum and maize in southern Honduras. Ceiba, v.29, n.2, p.353-361, 1988 .

PITRE, H.N. et al. A complex of lepidopterous defoliators on sorghum and maize in Honduras: some management tactis. Ceiba, v.38, n.2, p.109-119, 1997.

PORTILLO, H.E. et al. Langosta: a lepidopterous pest complex on sorghum and maize in Honduras. Florida Entomologist, v.74, n.2, p.287-296, 1991.

PORTILLO, H.E. et al. Oviposition preference of Spodoptera latifascia (Lepidoptera: Noctuidae) for sorghum, maize and noncrop vegetation. Florida Entomologist, v.79, n.4, p.552-562, 1996.

REUNIÃO DE PESQUISA DE SOJA DA REGIÃO SUL, 28., 2000. Recomendações técnicas para a cultura de soja no Rio Grande do Sul e em Santa Catarina 2000/2001. Santa Maria : UFSM/CCR/Departamento de Defesa Fitossanitária, 2000. 148p.

SANTOS, G.P.; COSENZA, G.W.; ALBINO, J.C. Biologia de Spodoptera latifascia (Walk., 1856) (Lepidoptera: Noctuidae) sobre folhas de eucalipto. Revista Brasileira de Entomologia, v.24, n.2, p.153-155, 1980.

SILVA, A.G.A. et al. Quarto catálogo dos insetos que vivem nas plantas do Brasil, seus parasitos e predadores. Rio de Janeiro : Ministério da Agricultura, 1968. Tomo 1, Parte II, 622p.

SILVAIN, J.F.; LALANNE-CASSOU, B. Distinction entre Spodoptera latifascia (Walk.) et Spodoptera cosmioides (Walk.), bona species (Lepidoptera: Noctuidae). Revue Française d'Entomologie, v.19, p.95-97, 1997.

SILVEIRA NETO, S. et al. Manual de ecologia dos insetos. São Paulo : Agronômica Ceres, 1976. 419p. 